\title{
Ordering Artificial Intelligence Based Recommendations to Tackle the SDGs with a Decision-Making Model Based on Surveys
}

\author{
Sergio Alonso ${ }^{1, *(\mathbb{D}}$, Rosana Montes ${ }^{1}\left(\mathbb{D}\right.$, Daniel Molina ${ }^{2}{ }^{(}$, Iván Palomares ${ }^{1,3}$, Eugenio Martínez-Cámara ${ }^{2}$, \\ Manuel Chiachio ${ }^{4}{ }^{\circ}$, Juan Chiachio ${ }^{4}{ }^{(0}$, Francisco J. Melero ${ }^{1}$, Pablo García-Moral ${ }^{5}$, Bárbara Fernández ${ }^{6}$, \\ Cristina Moral ${ }^{6}$, Rosario Marchena ${ }^{6}$, Javier Pérez de Vargas ${ }^{7}$ and Francisco Herrera ${ }^{2,7}$ (1)
}

Citation: Alonso, S.; Montes, R.;

Molina, D.; Palomares, I.;

Martínez-Cámara, E.; Chiachio, M.;

Chiachio, J.; Melero, F.J.; García-

Moral, P.; Fernández, B.; et al.

Ordering Artificial Intelligence Based

Recommendations to Tackle the SDGs with a Decision-Making Model Based on Surveys. Sustainability 2021, 13, 6038. https://doi.org/10.3390/ su13116038

Academic Editors: Álvaro Herrero, Carlos Cambra, Carlos Alonso de Armiño, Paweł Ksieniewicz and Paolo Gastaldo

Received: 21 April 2021

Accepted: 21 May 2021

Published: 27 May 2021

Publisher's Note: MDPI stays neutral with regard to jurisdictional claims in published maps and institutional affiliations.

Copyright: (C) 2021 by the authors Licensee MDPI, Basel, Switzerland. This article is an open access article distributed under the terms and conditions of the Creative Commons Attribution (CC BY) license (https:/ / creativecommons.org/licenses/by/ $4.0 /)$.
1 Department of Software Engineering, Andalusian Research Institute in Data Science and Computational Intelligence (DaSCI), University of Granada, 18071 Granada, Spain; rosana@ugr.es (R.M.); ivanpc@ugr.es (I.P.); fjmelero@ugr.es (F.J.M.)

2 Department of Computer Science and Artificial Intelligence, Andalusian Research Institute in Data Science and Computational Intelligence (DaSCI), University of Granada, 18071 Granada, Spain; dmolina@decsai.ugr.es (D.M.); emcamara@decsai.ugr.es (E.M.-C.); herrera@decsai.ugr.es (F.H.)

3 Department of Computer Science and Information Engineering, National Cheng Kung University, Tainan 70101, Taiwan

4 Department of Structural Mechanics and Hydraulic Engineering, Andalusian Research Institute in Data Science and Computational Intelligence (DaSCI), University of Granada, 18071 Granada, Spain; mchiachio@ugr.es (M.C.); jchiachio@ugr.es (J.C.)

5 Faculty of Arts, University of Granada, 18071 Granada, Spain; pablogarciamoral@gmail.com

6 Ferrovial S.A., C/ Principe de Vergara 135, 28002 Madrid, Spain; barbara.fernandez@ferrovial.com (B.F.); cmoral@ferrovial.com (C.M.); rmarchena@ci3.es (R.M.)

7 Royal Academy of Engineering of Spain (RAES), 28005 Madrid, Spain; jpvargas@raing.es

* Correspondence: zerjioi@ugr.es

Abstract: The United Nations Agenda 2030 established 17 Sustainable Development Goals (SDGs) as a guideline to guarantee a sustainable worldwide development. Recent advances in artificial intelligence and other digital technologies have already changed several areas of modern society, and they could be very useful to reach these sustainable goals. In this paper we propose a novel decision making model based on surveys that ranks recommendations on the use of different artificial intelligence and related technologies to achieve the SDGs. According to the surveys, our decision making method is able to determine which of these technologies are worth investing in to lead new research to successfully tackle with sustainability challenges.

Keywords: Sustainable Development Goals; artificial intelligence; emerging digital technologies; decision making

\section{Introduction}

The Sustainability Development Goals (SDGs) were adopted by the United Nations as a blueprint to achieve a global development for a more sustainable future for all people, reducing the poverty, protecting the environment, and producing global peace and prosperity [1].

Artificial intelligence (AI) and other digital technologies, such as big data, virtual and augmented reality, blockchain, etc. are strongly impacting in our lives [2,3], producing a continuous transformation in the so-called Fourth Industrial Revolution [4], and changing many fields in our daily life due to its versatility. Thus, AI can be foreseen to have a similar impact to tackle these SDGs $[5,6]$.

In a recent work [5], after a comprehensive literature review, the role that AI and digital technologies could play in the different SDGs was presented, and it provides several recommendations on how these technologies can help to achieve each of the SDGs. Due 
to the social nature of the SDGs and in order to improve the involvement of a committed citizenry, publicizing the recommendations is of interest so as to know how society would prioritize their implementation.

In this paper we have designed a decision making model which allows us to know and rank the preferred recommendations for the different SDGs. In doing so, we have conducted public surveys describing and proposing a set of candidate recommendations for each SDG. The respondents, composed by experts and interested people, selected the ones they consider most appropriate to achieve the goals for a particular SDG. Finally, an analysis is performed for each SDG along with an overall analysis evaluating the preferred technologies considering all the SDGs as a whole.

The remaining sections of the paper are as follows: Section 2 describes in the different SDGs and the AI and related technologies that are used as the recommendations (alternatives) for each one of the surveys. Section 3 presents the underlying preference representation format that is used in our decision making model (fuzzy preference relations) as well as our data acquisition scheme with surveys comparing alternatives in a pairwise fashion and finally, the decision-making model and its computation details. In Section 4, an analysis of the results is carried out and some conclusions for each one of the SDGs are presented. Section 5 presents some global results on the use of AI and related technologies for the SDGs as a whole. Finally, the main conclusions are summarized in Section 6.

\section{Sustainable Development Goals and Artificial Intelligence}

In this section we present a brief description of the 17 SDGs proposed by the United Nations Agenda 2030 [1] and some AI techniques and related technologies that can be helpful to attain the different SDGs.

\subsection{Sustainable Development Goals}

This section presents the United Nations' SDGs providing a categorization considering three broader dimensions of sustainability and six perspectives of human needs. Such a categorization was originally proposed in [6].

The 17 Sustainable Development Goals (SDGs) are part of the 2030 Agenda for sustainable development, and their objective is to serve as a global blueprint agenda to bring prosperity to people. They include crucial issues such as eradicating hunger and poverty, overcoming inequalities, various actions to guarantee a sustainable development tightly aligned with a healthful and renewable environment, and ensuring a maintainable development for future generations, amongst other global concerns.

Given their inherently transversal nature, the SDGs jointly take into account both economy needs (with particular focus on least developed countries or regions), social needs (such as access to education and equality), and environmental needs (concerned not only with the preservation of natural areas and their wildlife, but also with the mitigation of climate change). The 17 goals are summarized in Table 1.

The clear alignment among the SDGs and different aspects of economy, society and the environment, has given rise to various categorizations of them across disciplines. For instance the ones suggested by Vinuesa et al. [7] and $\mathrm{Wu}$ et al. [6]. In this paper we built upon the taxonomy proposed by Wu et al. in [6] (as illustrated in Figure 1), which consists of:

i. A first axis that discerns three dimensions or areas of human needs that require being fulfilled to achieve sustainability: economic, social and environmental.

ii. A second axis where each the above three dimensions is subdivided into two perspectives. 
Table 1. The 17 Sustainable Development Goals (SDGs). Source [1].

\section{Sustainable Development Goals}

\section{Goal 1: No poverty}

End poverty in all its forms everywhere.

Goal 2: Zero hunger

End hunger, achieve food security and improved nutrition and promote sustainable agriculture.

Goal 3: Good health and well-being

Ensure healthy lives and promote well-being for all at all ages.

Goal 4: Quality education

Ensure inclusive and equitable quality education and promote lifelong learning opportunities for all.

Goal 5: Gender equality

Achieve gender equality and empower all women and girls.

\section{Goal 6: Clean water and sanitation}

Ensure availability and sustainable management of water and sanitation for all.

\section{Goal 7: Affordable and clean energy}

Ensure access to affordable, reliable, sustainable and modern energy for all.

Goal 8: Decent work and economic growth

Promote sustained, inclusive and sustainable economic growth, full and productive employment and decent work for all.

\section{Goal 9: Industry, innovation and infrastructure}

Build resilient infrastructure, promote inclusive and sustainable industrialization and foster innovation.

\section{Goal 10: Reduced inequalities}

Reduce inequality within and among countries.

\section{Goal 11: Sustainable cities and communities}

Make cities and human settlements inclusive, safe, resilient and sustainable.

\section{Goal 12: Responsible consumption and production}

Ensure sustainable consumption and production patterns.

\section{Goal 13: Climate action}

Take urgent action to combat climate change and its impacts.

\section{Goal 14: Life below water}

Conserve and sustainably use the oceans, seas and marine resources for sustainable development.

\section{Goal 15: Life on land}

Protect, restore and promote sustainable use of terrestrial ecosystems, sustainably manage forests, combat desertification, and halt and reverse land degradation and halt biodiversity loss.

\section{Goal 16: Peace, justice and strong institutions}

Promote peaceful and inclusive societies for sustainable development, provide access to justice for all and build effective, accountable and inclusive institutions at all levels.

\section{Goal 17: Partnerships for the goals}

Strengthen the means of implementation and revitalize the global partnership for sustainable development.

Therefore, the following three dimensions and six perspectives of human needs are yielded (see Figure 1) [5]:

- Economic dimension: concentrated on sustainability and individual welfare at an economic level and considering the prosperity and welfare of the individuals. The two perspectives under this dimension are: (i) life, related to reducing poverty and ensuring food sustenance and health; (ii) economic and technological development, focused on economic growth, sustainable industrialization and innovation.

- Social dimension: focused on sustainable development with regard to equality, welfare and prosperity at community level. The two perspectives under this dimension are: (i) social development, concerning sustainable communities, peace, justice and global partnerships; (ii) equality in access to work and education, gender, socio-economic status, etc. 
- Environmental dimension: related to safeguarding and preserving the environment, as well as the sustainable management of its resources. The two perspectives under this dimension are: (i) resources, in the matter of water, clean energy, responsible production and consumption; (ii) natural environment, in connection with climate, land and underwater ecosystems.

It is worth highlighting that in [5], we reallocated SDG 14 (life below water), originally classified under the resources perspective in [6], into the natural environment perspective.
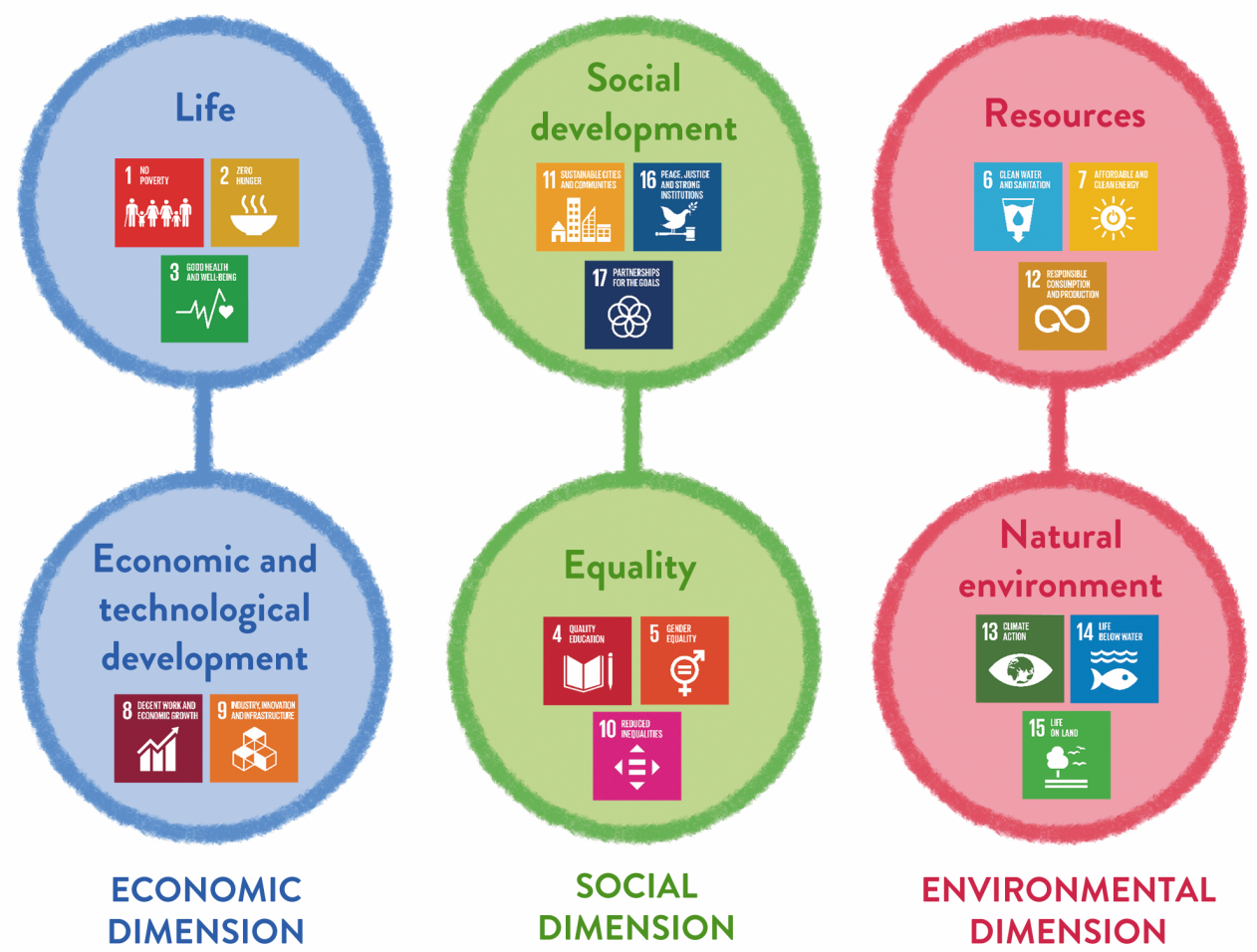

Figure 1. Classification of the SDGs into three dimensions and six perspectives of human needs. Source [5].

\subsection{Artificial Intelligence and Digital Technologies}

AI was first defined by John McCarthy in [8] as "the science and engineering of making intelligent machines, especially intelligent computer programs.", defining intelligence as "the computational part of the ability to achieve goals in the world". From a more pragmatic point of view, AI can be defined as the skill to solve problems that traditionally have been better worked out by humans, usually because it implies [9]:

- Natural language processing to be able to communicate in human language.

- Computer Vision, to process visual information.

- Knowledge representation, to represent all required information to take decisions in a reasonable way.

- Automated reasoning, to be able to process knowledge stored to perform specific actions and/or answer questions based on the input data and previous knowledge.

- Machine Learning, to allow the system to learn from examples and extract common patterns from the available information. This allows it to adapt its decisions/behavior to new scenarios. These techniques are quite interesting as there are many real-world problems that cannot be easily represented as symbolic rules but they can be learnt by processing a set of observations and extracting patterns and thus, being able to process future observations. 
The growing influence of $\mathrm{AI}$ in our daily lives raises concerns about the justification or motivation behind the decisions taken by AI algorithms that could affect us, in order to prove that the algorithms do not produce discrimination over specific collectives or take decisions over unjustified reasons. Accordingly, the development of explainable AI methods is useful for enhancing the utility and the interpretability of the decisions of AI algorithms. Hence, explainable AI is an additional challenge to the current one related to Fairness, Accountability, Transparency and Ethics (FATE). The aim of providing to AI systems those features is the development of trustworthy AI, which is featured by:

- Robustness: the AI system must work correctly avoiding any damages.

- Lawfulness: respecting all laws and required regulations.

- Ethicalness: respecting principles as human dignity, individual freedom, justice, equality, non-discrimination or citizen rights, among others.

Because AI systems need an adequate flow of data to learn, it usually requires the support of other digital technologies for extracting, storing, processing and receiving the data. In the following, we briefly describe some of those as they are considered for the different proposals and recommendations in the study:

- Internet of Things (IoT). It refers to the fact of having several interconnected devices, capable not only to monitor data from sensors but also to do some simple processing over it and to send the information using the Internet.

- Blockchain. It allows the exchange of data in blocks guarantying the immutability of the data, and the traceability of new data blocks. Blockchain is able to define secure administration of data over a distributed network [10].

- Augmented Reality and Virtual Reality. Virtual reality is a computational simulation of a real system that enables a user to perform operations on the simulated system in a similar way than in reality. While in virtual reality the user is immersed in the virtual simulation, in augmented reality there is a merging of the virtual model with the real one (real and synthetic images are merged, or information about the real world is merged in a video stream).

- Digital twin. In this technology a real object is represented with a digital model, called twin, with a real time communication among them in order to ensure a trustworthy digital representation. This allow to study the real object through the digital one.

- $5 \mathrm{G}$ communication infrastructure. This is the 5 th generation of mobile communication technologies, and it is remarkable for its great capacity to connect millions of devices with a fast and reliable communication between them.

- Smart city. A smart city is an urban area that uses different devices and sensors to collect data that will be used to improve the experience of the citizens across the city.

- Smart territories. These refer to the technology used for urban and rural data management beyond the scope of smart cities.

- Big Data. These are the technologies designed for processing a high volume and variety of data at high velocity. It is crucial when the data are generated with a high frequency [11].

- Recommender and Information Systems. These systems ease the processes of choosing among different alternatives and provide quality information for decision makers.

\section{Materials and Methods: Decision Making Model for Ranking AI Based Recommendations}

In this section we describe our approach to develop a decision making model based on surveys that ranks different $\mathrm{AI}$ and related digital technologies in order to achieve the SDGs. We first describe some basic information about the information structure that is used to manage our data, that is, preference relations. Then we describe the tools that we have used in order to create the surveys that we have conducted. Finally, we describe the novel decision making model that ranks the different AI and related technologies that can be applied to achieve the SDGs from the data collected in the surveys. 


\subsection{Preferences Expression}

There are several aspects to take into account in a decision process to choose the best alternative from a set of them. One of this aspects is how the preferences information is expressed [12]. Several options can be found in the literature as preference orderings (the alternatives are ranked according to their goodness) [13], utility functions (each alternative is given an utility evaluation using a particular scale) [14] or preference relations, where each pair on the set of alternatives are compared one against the other [15,16]. Moreover, we can find different decision making models where different preference representation structures can be simultaneously used $[17,18]$. In this paper we focus on the use of fuzzy preference relations $[15,19]$ :

Definition 1. A fuzzy preference relation $P$ on a set of alternatives $X=\left\{x_{1}, \ldots, x_{n}\right\}$ is a fuzzy set on the product set $X \times X$, i.e., it is characterized by a membership function $\mu_{P}: X \times X \rightarrow[0,1]$.

When the number of alternatives is small the fuzzy preference relation can be easily represented by a $n \times n$ matrix: $P=\left(p_{i j}\right)$ where each $p_{i j}=\mu_{P}\left(x_{i}, x_{j}\right)(\forall i, j \in X)$ indicates the degree of preference of the alternative $x_{i}$ over $x_{j}$ :

- $p_{i j}=1$ means that alternative $x_{i}$ is absolutely preferred to $x_{j}$,

- $\quad p_{i j}=0.5$ means equal preference for $x_{i}$ and $x_{j}\left(x_{i} \sim x_{j}\right)$, and

- $\quad p_{i j}>0.5$ means that $x_{i}$ is preferred to $x_{j}\left(x_{i} \succ x_{j}\right)$.

One of the advantages of using fuzzy preference relations is that they can represent the preferences of decision makers in a realistic way, including missing information $[20,21]$ and even contradictions and inconsistencies [22,23].

However, asking decision makers to directly provide a fuzzy preference relation that summarizes his/her opinions on the alternatives may be difficult, because their lack of familiarity with the representation format and the large number of comparisons among pairs of alternatives, specially if the number of available alternatives is high. Accordingly, we present a multi-person decision making model based on surveys that uses preference relations as its inner preference structure to overcome that challenge.

\subsection{Data Acquisition}

The design or adaptation of instruments for data collection in scientific research is a very common phase for conducting a study to accept or reject research questions or hypotheses. Questionnaires are one of the most used data collection tools, along with interviews and observation. A desirable property for quality in a questionnaire is the ability to measure the variables for which it was designed, that it is, its validity. Another desirable property is to be flexible enough for the problem being considered. A third property is oriented to its availability to reach more people. In this regard, using online digital media to support questionnaires significantly helps reaching more people, reducing answer time and also reducing costs.

We can distinguish two types of questionnaires: (i) open, those that present open or flexible answers for the respondent, and (ii) closed, those that only allow to choose one option from a fixed set of choices. Closed surveys are the most widely used because they are the easiest to use, especially in digital contexts. Salganik and Levi's work on Wiki Surveys [24] proposes the free-software online platform https://allourideas.org (accessed date: 24 May 2021) that allows handling uncertainty in the answers (although their model does not integrate it), and it allows to build and distribute surveys with open and closed questions. Another interesting feature of the referred platform is that it allows randomization in the answers: The compared alternatives are randomly selected among the set of alternatives and can appear as the left or right option. This randomization helps preventing biases stem from linguistic and cultural effects. 
The advantage provided by the allourideas.org platform is that it allows us to have a Multi-person Decision Making model that collects simple preference values about pairs of alternatives (a widely used methodology to gather social data [25]), just by a single click on a button instead of expressing a numerical value used as in a fuzzy preference relation. Its interface provides three buttons: one to express preference for the alternative on the left, other to express preference for the alternative on the right, and a third button may be used when the decision maker is unsure about his/her preference. That is, the decision makers can express uncertainty in their answers for various reasons, which are shown in Figure 2. This proposed survey is simpler and faster than other Decision Making approximations to gather opinions. In addition, since the system handles uncertainty as a possible answer it helps to deal with missing information: When the system gathers enough answers from different persons the problem of missing values is alleviated.

\section{I can't decide because...}
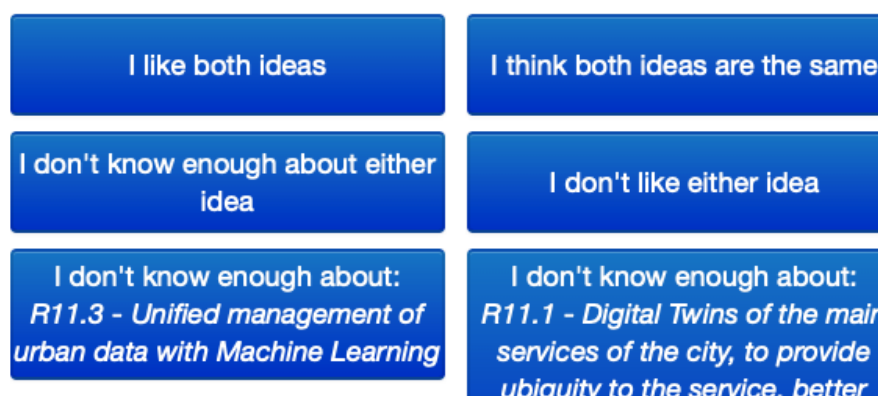

I don't like either idea

I don't know enough about:

R11.1 - Digital Twins of the main

services of the city, to provide

ubiquity to the service, better

inform the citizen, and monitoring

social impact

I just can't decide

Figure 2. Hesitation choices provided by the platform when the respondent is not able to vote to an option.

We have used this tool to carry out 17 different surveys, one per SDG, which allow us to assess which proposals related to AI and digital technologies are seen by the experts as the most relevant. The studied proposals are listed in Section 4, which were extracted from [26]. In that work, a comprehensive literature review process was carried out to investigate the use of $\mathrm{AI}$ and digital technologies as enablers for the fulfillment of the SDGs. From that study a SWOT analysis was made for each SDGs regarding the use of AI and digital technologies followed by an analysis of the collection of the provided recommendations. Those recommendations are the ones being analyzed in this study.

Our methodological approach consisted of creating a website with explanatory texts and videos on the SDGs and the proposed actions, followed by a link to the survey. These surveys were conducted anonymously, and it was not necessary to provide information characterizing the expert. However, favored by the strong presence of RAI and Ferrovial in social networks, an invitation campaign to visit the website was launched, reaching the academic and professional engineering sector at worldwide level. In addition, we also contacted associations with strong links to certain SDGs. For example, the Spanish Association for Artificial Intelligence (AEPIA), the Andalusian Institute for Earth System Research (IISTA), the Civil Engineers Association, the Spanish Association for Environmental Education, and specialists in Climatology and Hydrogeography. Table 2 provides information regarding the collected data from the surveys initiated in December 2020 and closed in January 2021.

To ease the access to the surveys and provide enough information to the respondents about the different SDGs and the proposed AI and related technologies we prepared the 
website http: / / dasci.es/ia4ods in which we provide a brief description for each one of the SDGs, the available alternatives for this study (for each SDG) and the links to the different surveys. Moreover, raw data collected from these surveys, along with the scripts to process it, are accessible at https: / / github.com/ari-dasci/S-SDG-Decision.

Table 2. Number of responses received in each survey for the period of two months (data collected from the surveys on 1 February 2021). \#Votes correspond to the evaluations in which an alternative was preferred to another or both where equally liked or disliked (like_both or dislike_both). \#Unknown sums the number of times in which any of the alternatives was marked as unknown by the respondent.

\begin{tabular}{clcc}
\hline SurveyID & URL & \#Votes & \#Unknown \\
\hline SDG-1 & http://allourideas.org/SDG1 & 481 & 31 \\
\hline SDG-2 & http://allourideas.org/SDG2 & 290 & 2 \\
\hline SDG-3 & http://allourideas.org/SDG3 & 310 & 0 \\
\hline SDG-4 & http://allourideas.org/SDG4 & 251 & 1 \\
\hline SDG-5 & http://allourideas.org/SDG5 & 148 & 1 \\
\hline SDG-6 & http://allourideas.org/SDG6 & 133 & 36 \\
\hline SDG-7 & http://allourideas.org/SDG7 & 113 & 8 \\
\hline SDG-8 & http://allourideas.org/SDG8 & 238 & 0 \\
\hline SDG-9 & http://allourideas.org/SDG9 & 193 & 0 \\
\hline SDG-10 & $\mathrm{http://allourideas.org/SDG10}$ & 175 & 0 \\
\hline SDG-11 & $\mathrm{http://allourideas.org/SDG11}$ & 174 & 5 \\
\hline SDG-12 & $\mathrm{http://allourideas.org/SDG12}$ & 169 & 0 \\
\hline SDG-13 & $\mathrm{http://allourideas.org/SDG13}$ & 180 & 0 \\
\hline SDG-14 & $\mathrm{http://allourideas.org/SDG14}$ & 180 & 0 \\
\hline SDG-15 & $\mathrm{http://allourideas.org/SDG15}$ & 172 & 4 \\
\hline SDG-16 & $\mathrm{http://allourideas.org/SDG16}$ & 179 & 1 \\
\hline SDG-17 & $\mathrm{http://allourideas.org/SDG17}$ & 180 & 2 \\
\hline
\end{tabular}

\subsection{A Preference Relation Based Decision-Making Model for Recommendations Ranking}

Once we have collected enough answers for each survey, a collection of crisp comparisons over all the pairs of alternatives is obtained. At this point we have to aggregate all the information in order to determine which alternatives are preferred over the others. To do so, we compute a fuzzy preference relation $P^{o}$ for each SDG $(o \in\{1, \ldots, 17\})$ as follows:

1. Initialize $P^{o}$, setting $p_{i k}^{o}=0$.

2. For each respondent stating that alternative $x_{i}^{o}$ is preferred over $x_{k}^{o}$, increment $p_{i k}^{o}$ : $p_{i k}^{o} \leftarrow p_{i k}^{o}+1$.

3. For each respondent stating that alternatives $x_{i}^{o}$ and $x_{k}^{o}$ are equally preferred (either using like_both or dislike_both) increment both $p_{i k}^{o}$ and $p_{k i}^{o}: p_{i k}^{o} \leftarrow p_{i k}^{o}+0.5$ and $p_{k i}^{o} \leftarrow p_{k i}^{o}+0.5$.

4. For each preference value $p_{i k^{\prime}}^{o}$, normalize it to the unit interval $[0,1]: p_{i k}^{o}=\frac{p_{i k}^{o}}{p_{i k}^{o}+p_{k i}^{o}}$.

We remark that the presented method obtains fuzzy preference relations that comply with additive reciprocity [27], that is, $p_{i k}^{o}+p_{k i}^{o}=1$, which is usually a desirable property. Let's clarify the proposed method with two illustrative examples: 
- Let's assume that two respondents stated that $x_{2}^{1}$ is preferred over $x_{3}^{1}$ and one additional one stated that $x_{3}^{1}$ is preferred over $x_{2}^{1}$. Then we would have two positive votes for $x_{2}^{1}$ and one for $x_{3}^{1}$ and its final $p_{23}^{1}$ and $p_{32}^{1}$ values would be: $p_{23}^{1}=\frac{2}{2+1}=0.67$ and $p_{32}^{1}=\frac{1}{1+2}=0.33$.

- Let's assume that two respondents stated that $x_{3}^{3}$ is preferred over $x_{1}^{3}$, another one stated that $x_{1}^{3}$ is preferred over $x_{3}^{3}$, and a third one equally disliked both $x_{1}^{3}$ and $x_{3}^{3}$. Then we would have $p_{13}^{3}=\frac{1.5}{1.5+2.5}=0.375$ and $p_{31}^{3}=\frac{2.5}{2.5+1.5}=0.625$.

Once we have obtained the fuzzy preference relations $P^{o}$, we can also compute their additive consistency level [22] $C P^{o} \in[0,1]$. The consistency level measures how well the fuzzy preference relation complies with additive transitivity [27]:

$$
\left(p_{i j}^{o}-0.5\right)+\left(p_{j k}^{o}-0.5\right)=\left(p_{i k}^{o}-0.5\right)
$$

This is usually a desirable property as it implies no contradictions in the preferences expressed in the relation. Specifically, $C P^{o}=1$ means that the fuzzy preference relation is completely consistent whilst $C P^{o}=0$ means that the relation is completely inconsistent.

To decide which alternative is preferred among the others for any alternative $x_{i}^{o}$, we can compute a Quantifier Guided Dominance Degree $\left(Q G D D_{i}^{o}\right)$ and a Quantifier Guided Non Dominance Degree $\left(Q G N D D_{i}^{o}\right)$ [17]. The first one measures in a $[0,1]$ scale how $x_{i}^{o}$ dominates all the others in a fuzzy majority sense. The later provides a degree (also in a $[0,1]$ scale) in which $x_{i}^{o}$ is not dominated by a fuzzy majority of the remaining alternatives. In our case the adopted fuzzy quantifier to compute these measures is "most" with the pair $(0.3,0.8)$. The interested reader is referred to [17] and the supplementary material of this paper for more implementation details.

Lastly, from the previous degrees we can order the alternatives for each $S D G^{\circ}$ applying the following procedure:

1. Order the alternatives according to their $Q G D D_{i}^{o}$.

2. For each two consecutive alternatives $x_{i}^{o}$ and $x_{k}^{o}$ : if $Q G D D_{i}^{o}-Q G D D_{k}^{o}<0.03$ and $Q G N D D_{i}^{o}-Q G N D D_{k}^{o}<-0.03$ then swap $x_{i}^{o}$ ad $x_{k}^{o}$. That is: if two alternatives have a very similar QGDD, reorder them according to their QGNDD (unless the QGNDD are also very similar).

3. For each two consecutive alternatives $x_{i}^{o}$ and $x_{k}^{o}$ if $\left|Q G D D_{i}^{o}-Q G D D_{k}^{o}\right|<0.03$ and $\left|Q G N D D_{i}^{o}-Q G N D D_{k}^{o}\right|<0.03$ then $x_{i}^{o} \sim x_{k}^{o}$ (both alternatives are equally preferred), otherwise $x_{i}^{o} \succ x_{k}^{o}\left(x_{i}^{o}\right.$ is preferred to $\left.x_{i}^{o}\right)$.

This procedure orders the alternatives according to their QGDD: If two alternatives have very different QGDD (more than 3\%) the one with bigger QGDD is preferred to the other. If both alternatives have similar QGDD (less than 3\% difference), then we check the difference among their QGNDD. If the difference is large enough (again 3\%), we select the one with the largest QGNDD. If both alternatives have very similar QGDD and QGNDD, then the two alternatives are considered equally preferred.

\section{Results and Discussion: Ordering Recommendations Based on AI and Digital Technologies}

In this section we present the results of the decision-making procedure applied to the rank proposed recommendations for every SDG. For each $S D G^{g}, g \in\{1, \ldots, 17\}$, we provide:

- The fuzzy preference relation $P^{g}$ obtained from the decision making process described in Section 3.3.

- Its corresponding consistency level $C P^{g}$.

- The total number of pairwise evaluations made by the experts $T E^{g}$, distinguishing between the evaluations in which one option is preferred and those where both alternatives are equally liked or disliked. 
- A table showing the QGDD and QGNDD for each alternative, and the number of times each alternative was "not known" by the respondents.

- The final preference ordering for the alternatives.

- A brief discussion about the results yielded from an SDG vantage point.

Finally, we take an overall look at all the rankings to identify the most preferred AI techniques and related technologies that can be applied to the SDGs as a whole.

\subsection{SDG 1: No Poverty}

The proposed recommendations for this SDG (see Table 3) were among the ones with the highest participation rates. Its associated alternatives' QGDDs range between 0.59 for the most preferred alternative and 0.45 for the least preferred one, which indicates no signs of very strong preference for -or against- the alternatives, thus having a moderately balanced distribution of individual votes. Nevertheless, there are sufficient differences to distinguish between the three options without incurring in cases of equal preference for any two of them. Transparent e-governance decision-making processes founded on blockchain (R1.1) constitute the most preferred recommendation to fight poverty, arguably due to the public perceived potential for mitigating institutional corruption (which is generally a related factor to poverty in many nations). The second most preferred option (R1.2) stimulates the construction of accessible large-scale Big Data infrastructures, which facilitate data acquisition and sharing underpinned by Internet-of-Things and sensor technologies to collect data for measuring and identifying poverty; followed lastly by automation strategies committed to economic safety of employees and jobs (R1.3).

Table 3. Proposed actions for SDG 1 involving the use of AI and digital technologies.

Goal 1: No poverty.

R1.1-Governance based on blockchain for transparent decision-making against poverty.

R1.2-Realistic, objective and universal measurement of poverty guided by Big Data.

R1.3-Automation committed to employee's economic safety underpinned by education and innovation.

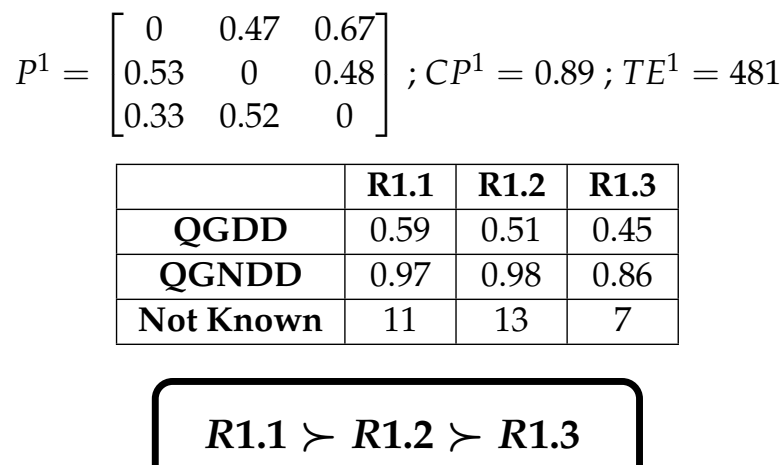

\begin{tabular}{|c|c|c|c|}
\hline & R1.1 & R1.2 & R1.3 \\
\hline QGDD & 0.59 & 0.51 & 0.45 \\
\hline QGNDD & 0.97 & 0.98 & 0.86 \\
\hline Not Known & 11 & 13 & 7 \\
\hline
\end{tabular}

\subsection{SDG 2: Zero Hunger}

From the five proposed recommendations to pursue the targets underlying this goal (see Table 4), there is a clear preference for three of them (R2.4, R2.1 and R2.5), with almost no discernible difference between their dominance scores. This suggests that AI solutions combining and balancing the following three strategies would be perceived as popular holistic solutions: (i) effective hunger risk management through early warning systems (R2.4), (ii) open shared data platforms to enhance smart agriculture and food production (R2.1); and (iii) helping the population acquire sustainable and healthier nourishment habits, e.g., via personalization (R2.5). By contrast, R2.3 (traceable food production and supply via blockchain) and R2.2 (governance and big data-driven decisions to ensure food 
sustenance) are comparatively less voted alternatives. Almost no uncertain votes have been reported for this SDG.

Table 4. Proposed actions for SDG 2 involving the use of AI and digital technologies.

Goal 2: Zero hunger.

R2.1-Accessible platforms for data integration in agricultural-food production.

R2.2-Equality-aware decisions for food sustenance through governance and Big Data.

R2.3-Traceable food production and supply under AI and blockchain.

R2.4-Early Warning Systems to anticipate to risks in safe food sustenance.

R2.5-Promoting sustainable, healthy and responsible eating across the population.

$\begin{aligned} & P^{2}=\left[\begin{array}{ccccc}0 & 0.65 & 0.5 & 0.25 & 0.71 \\ 0.35 & 0 & 0.4 & 0.41 & 0.35 \\ 0.5 & 0.6 & 0 & 0.43 & 0.19 \\ 0.75 & 0.59 & 0.57 & 0 & 0.55 \\ 0.29 & 0.65 & 0.81 & 0.45 & 0\end{array}\right] ; C P^{2}=0.84 ; T E^{2}=290 \\ & \begin{array}{|c|c|c|c|c|c|}\hline & \mathbf{R 2 . 1} & \mathbf{R 2 . 2} & \mathbf{R 2 . 3} & \mathbf{R 2 . 4} & \mathbf{R 2 . 5} \\ \hline \text { QGDD } & 0.59 & 0.38 & 0.48 & 0.6 & 0.59 \\ \hline \text { QGNDD } & 1.0 & 0.76 & 0.94 & 1.0 & 0.96 \\ \hline \text { Not Known } & 1 & 0 & 0 & 0 & 1 \\ \hline\end{array}\end{aligned}$

$R 2.4 \sim R 2.1 \succ R 2.5 \succ R 2.3 \succ R 2.2$

\subsection{SDG 3: Good Health and Well-Being}

There is a strict order of preference between the five proposed alternatives (see Table 5) to achieve good health and well-being for all. Trustworthy and explainable AI systems (R3.1) amply position themselves as the most popular research and innovation trend in the health landscape, followed by the urging need to foster digital training by professionals for achieving an optimal human-AI trade-off (R3.3). The homogenization of health data (R3.2) and the deployment of smart cities/territories to control epidemic diseases (R3.4) are both similarly ranked as intermediate options. The lowest dominance is shown by (R3.5), "Personalized recommendations for healthy habits". This is not an unexpected outcome, since the state of affairs in health recommender systems beyond personalized patient healthcare-i.e., under the viewpoint of preventive health for the wider population-is still at its infancy, specially compared to the advances made in AI-guided medical diagnosis, prognosis, and so on. Given their great potential to avoid preventable diseases, more research is therefore needed to promote the application of personalized recommender systems in the health and well-being domain.

Table 5. Proposed actions for SDG 3 involving the use of AI and digital technologies.

\begin{tabular}{l}
\hline Goal 3: Good health and well-being. \\
\hline R3.1-Medical prediction and diagnosis through explainable trustworthy AI. \\
R3.2-Homogenization of multiple medical and health data sources. \\
R3.3-Technological training of health/medical professionals. \\
R3.4-Smart territories to improve health security detecting and controlling infectious diseases. \\
R3.5-Personalized recommendations for promoting healthy habits. \\
\hline
\end{tabular}




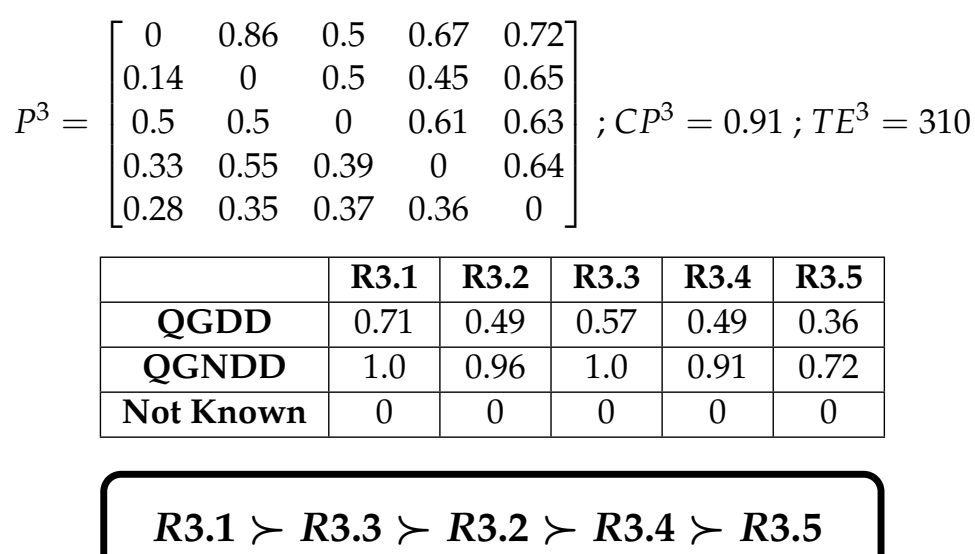

\begin{tabular}{|c|c|c|c|c|c|}
\hline & R3.1 & R3.2 & R3.3 & R3.4 & R3.5 \\
\hline QGDD & 0.71 & 0.49 & 0.57 & 0.49 & 0.36 \\
\hline QGNDD & 1.0 & 0.96 & 1.0 & 0.91 & 0.72 \\
\hline Not Known & 0 & 0 & 0 & 0 & 0 \\
\hline
\end{tabular}

\subsection{SDG 4: Quality Education}

Table 6 shows the recommendations for SDG 4. Two popular proposals can be observed. The first one is R4.3, "Open and free education with recommended contents to favour lifelong and ubiquitous learning", closely followed in terms of respondents preferences by R4.1, "AI for personalized learning and inclusive education". This is something expected, specially considering how important ubiquitous learning paradigms are nowadays, as evidenced by the COVID-19 pandemic. Also, the "inclusive education" is an important goal for many experts in learning. The next best valued proposal is R4.5, "Universal use of institutional frameworks that guarantee the treatment of information by any student with equity and security". Finally, the least preferred options are R4.2 "Use of virtual/augmented reality techniques and mobile apps to disseminate teaching contents, and support education under different learning styles" and R4.4, "Conversational assistants for academic management and quality evaluation of teaching received". They can be considered as innovative but less specific proposals. This is therefore a reasonable result, since these two options are more technically-focused, and less related to salient aspects of SDG 4 such as inclusive education.

Table 6. Proposed actions for SDG 4 involving the use of AI and digital technologies.

Goal 4: Quality education.

R4.1-AI for personalized learning and inclusive education.

R4.2-Use of virtual/augmented reality techniques and mobile apps to disseminate teaching contents, and support education under different learning styles.

R4.3-Open and free education with recommended contents to favour lifelong and ubiquitous learning.

R4.4-Conversational assistants for academic management and quality evaluation of teaching received.

R4.5-Universal use of institutional frameworks that guarantee the treatment of information by any student with equity and security.

$$
P^{4}=\left[\begin{array}{ccccc}
0 & 0.85 & 0.31 & 0.78 & 0.57 \\
0.15 & 0 & 0.21 & 0.6 & 0.44 \\
0.69 & 0.79 & 0 & 0.7 & 0.76 \\
0.22 & 0.4 & 0.3 & 0 & 0.37 \\
0.43 & 0.56 & 0.24 & 0.63 & 0
\end{array}\right] ; C P^{4}=0.9 ; T E^{4}=251
$$

\begin{tabular}{|c|c|c|c|c|c|}
\hline & R4.1 & R4.2 & R4.3 & R4.4 & R4.5 \\
\hline QGDD & 0.7 & 0.36 & 0.74 & 0.35 & 0.51 \\
\hline QGNDD & 1.0 & 0.71 & 1.0 & 0.69 & 0.94 \\
\hline Not Known & 0 & 0 & 0 & 0 & 1 \\
\hline
\end{tabular}




\section{$R 4.3 \succ R 4.1 \succ R 4.5 \succ R 4.2 \sim R 4.4$}

\subsection{SDG 5: Gender Equality}

Amongst the recommendations of this SDG (see Table 7), there are two highly preferred options, R5.4, "AI for the detection of denigrating or discriminatory content and behavior towards women and girls in social networks" and R5.2, "Development, and enforcement, of strong legislation in defense of women and girls", both centered in avoiding legal and social discrimination. They are followed by R5.3, "AI for gender bias detection in recruitment processes or deliberative bureaucracies", which is more oriented to discrimination in work environments. The least preferred option is R5.1, "Basic and specialized training in new technologies for girls and women", which suggests the promotion of STEM studies for girls and women to mitigate gender breaches across technical professions in the near future.

Table 7. Proposed actions for SDG 5 involving the use of AI and digital technologies.

\begin{tabular}{l}
\hline Goal 5: Gender equality. \\
\hline R5.1-Basic and specialized training in new technologies for girls and women. \\
R5.2-Development, and enforcement, of strong legislation in defense of women and girls. \\
R5.3-AI for gender bias detection in recruitment processes or deliberative bureaucracies. \\
R5.4-AI for the detection of denigrating or discriminatory content and behavior towards \\
women and girls in social networks.
\end{tabular}

$$
P^{5}=\left[\begin{array}{cccc}
0 & 0.33 & 0.35 & 0.28 \\
0.67 & 0 & 0.59 & 0.4 \\
0.65 & 0.41 & 0 & 0.52 \\
0.72 & 0.6 & 0.48 & 0
\end{array}\right] ; C P^{5}=0.94 ; T E^{5}=148
$$

\begin{tabular}{|c|c|c|c|c|}
\hline & R5.1 & R5.2 & R5.3 & R5.4 \\
\hline QGDD & 0.33 & 0.6 & 0.55 & 0.62 \\
\hline QGNDD & 0.67 & 0.99 & 0.99 & 1.0 \\
\hline Not Known & 0 & 0 & 1 & 0 \\
\hline
\end{tabular}

$$
R 5.4 \sim R 5.2 \succ R 5.3 \succ R 5.1
$$

\subsection{SDG 6: Clear Water and Sanitation}

From the five proposed recommendations to pursue some targets under this goal (see Table 8), there is a noticeably stronger preference for two of them: R6.1, "IoT technologies for monitoring all kinds of water facilities, networks and ecosystems" and R6.3, "Intelligent techniques for the prediction and detection of failures and breaks in water infrastructures, as well as the detection of variables or parameters for which there is no specific sensorization", with no significant difference between their dominance scores. Both have in common the use of sensors in water ecosystems to detect (or even predict) possible failures that could imply water leakage (or loss of quality). The next recommendation in popularity is R6.4, "Management and governance of water resources, infrastructures and ecosystems by using decision support systems, blockchain as well as virtual reality and augmented reality", in which the opportunities of these new technologies are positively valued. The last two options are R6.5, “Using machine learning techniques to improve prediction models of existing water systems", and R6.2, "Digital Twins on water infrastructures, water-linked ecosystems and contamination processes that allow their study, experimentation and dissemination". Arguably, the concept of digital twins might be comparatively less known within the scope of water systems, and therefore it would be perceived as less necessary 
than other proposed lines of action, as also occurs to a smaller extent with the techniques suggested in R6.4.

Table 8. Proposed actions for SDG 6 involving the use of AI and digital technologies.

\begin{tabular}{l}
\hline Goal 6: Clean water and sanitation. \\
\hline R6.1-IoT technologies for monitoring all kinds of water facilities, networks and ecosystems. \\
R6.2-Digital Twins on water infrastructures, water-linked ecosystems and contamination \\
processes that allow their study, experimentation and dissemination. \\
R6.3-Intelligent techniques for the prediction and detection of failures and breaks in water \\
infrastructures, as well as the detection of variables or parameters for which there is no \\
specific sensorization. \\
R6.4-Management and governance of water resources, infrastructures and ecosystems by \\
using decision support systems, blockchain as well as virtual reality and augmented reality. \\
R6.5-Using machine learning techniques to improve prediction models of existing water sys- \\
tems.
\end{tabular}

$$
P^{6}=\left[\begin{array}{ccccc}
0 & 0.71 & 0.58 & 0.67 & 0.65 \\
0.29 & 0 & 0.22 & 0.46 & 0.3 \\
0.42 & 0.78 & 0 & 0.55 & 0.71 \\
0.33 & 0.54 & 0.45 & 0 & 0.86 \\
0.35 & 0.7 & 0.29 & 0.14 & 0
\end{array}\right] ; C P^{6}=0.87 ; T E^{6}=133
$$

\begin{tabular}{|c|c|c|c|c|c|}
\hline & R6.1 & R6.2 & R6.3 & R6.4 & R6.5 \\
\hline QGDD & 0.66 & 0.31 & 0.65 & 0.54 & 0.36 \\
\hline QGNDD & 1.0 & 0.62 & 1.0 & 0.96 & 0.68 \\
\hline Not Known & 7 & 5 & 5 & 10 & 9 \\
\hline
\end{tabular}

$R 6.1 \sim R 6.3 \succ R 6.4 \succ R 6.5 \succ R 6.2$

\subsection{SDG 7: Affordable and Clean Energy}

From the different proposals in this SDG (see Table 9), there is a clear ranking among them. The most popular recommendation is R7.4, "Intelligent and robotic systems to reduce the inspection and maintenance costs of energy facilities", focused on using technologies to create more reliable energy facilities. The next one is R7.2, "High-fidelity models for long-term prediction of renewable energy resources (wind, solar irradiation, etc.)". This is an expected outcome because renewable energies are publicly considered as the energy of the future. The next one is R7.5, "Blockchain to control traceability and better monitoring of financial flows for investment in clean and renewable energy in developing countries", remarking the advantages of blockchain. The next one is R7.1, "Data compression and distributed computing technologies to reduce energy consumption in data storage and processing centers (data centers)" aimed at reducing the consumption of energy in data centers: a not widely tackled problem yet in spite of its increasing significance. The last one is R7.3, "Blockchain technology for the secure management of smart electrical grids", that enforces again the blockchain possibilities. It is also noteworthy that this is the SDG which received less answers in the study. 
Table 9. Proposed actions for SDG 7 involving the use of AI and digital technologies.

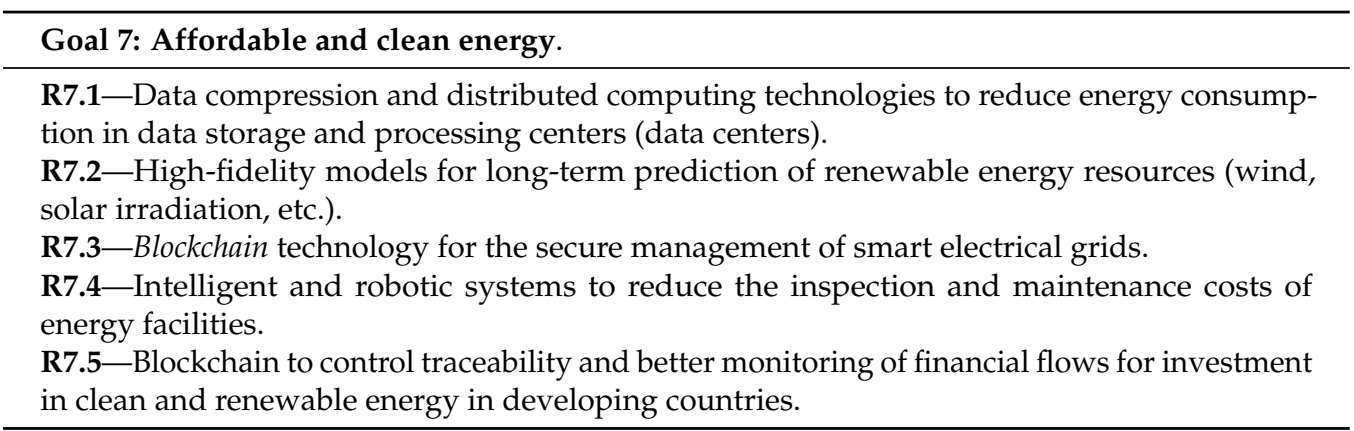

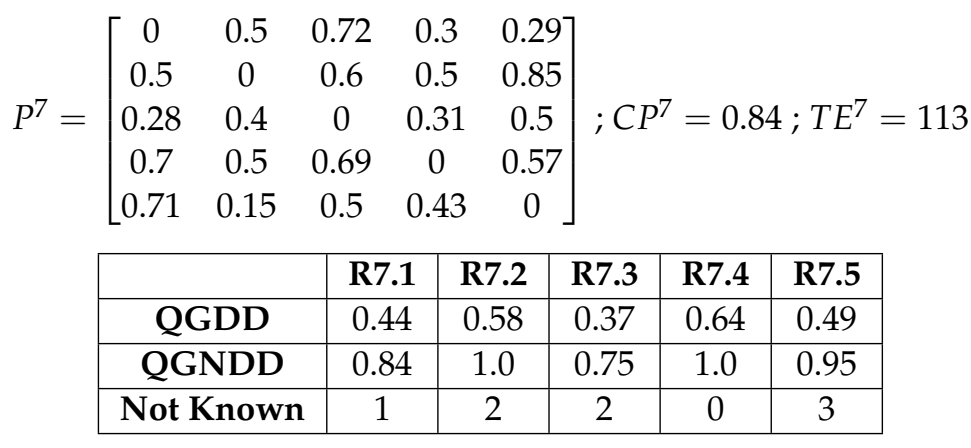

\section{$R 7.4 \succ R 7.2 \succ R 7.5 \succ R 7.1 \succ R 7.3$}

\subsection{SDG 8: Decent Work and Economic Growth}

This SDG (see Table 10) received a higher number of answers than for most of the other goals and, remarkably, no respondents marked none of the proposals as not known. From the answers, we can observe that the most positively perceived proposal is by far R8.1, "Updated education systems and fight against digital breach". The digital breach is clearly considered a big challenge, and the recommendations to update education systems have been significantly supported. The next one is R.8.4, "Ensure that raw materials for electronics and digital technologies do not come from slave labor", possibly because of the increased awareness of the economic systems underpinned by dubious principles and forced work. This is specially important in many developing countries which produce raw materials for many technological components. The third most valued option is R8.2, "Efficient and productive innovation for economic growth, with special emphasis on generating added value and new products and services", and the least popular one was R8.3, "Universal access to the internet and computers as a basis for access to banking services and other credit and tax processing services". In sum, knowledge acquisition through digitization in education systems (R8.1) is necessary and linked to digital inequality in all countries: overall this is one of the highest rated recommendations in this study from across all the 17 SDGs.

Table 10. Proposed actions for SDG 8 involving the use of AI and digital technologies.

Goal 8: Decent work and economic growth.

R8.1-Updated education systems and fight against digital breach.

R8.2-Efficient and productive innovation for economic growth, with special emphasis on generating added value and new products and services.

R8.3-Universal access to the internet and computers as a basis for access to banking services and other credit and tax processing services.

R8.4-Ensure that raw materials for electronics and digital technologies do not come from slave labor. 


$$
P^{8}=\left[\begin{array}{cccc}
0 & 0.85 & 0.79 & 0.7 \\
0.15 & 0 & 0.68 & 0.44 \\
0.21 & 0.32 & 0 & 0.44 \\
0.3 & 0.56 & 0.56 & 0
\end{array}\right] ; C P^{8}=0.92 ; T E^{8}=238
$$

\begin{tabular}{|c|c|c|c|c|}
\hline & R8.1 & R8.2 & R8.3 & R8.4 \\
\hline QGDD & 0.8 & 0.49 & 0.35 & 0.54 \\
\hline QGNDD & 1.0 & 0.88 & 0.69 & 0.97 \\
\hline Not Known & 0 & 0 & 0 & 0 \\
\hline
\end{tabular}

$$
R 8.1 \succ R 8.4 \succ R 8.2 \succ R 8.3
$$

\subsection{SDG 9: Industry, Innovation and Infrastructure}

There are five proposals in the SDG related to industry, innovation and infrastructure, as shown in Table 11. It can be observed significant differences in the ranking or preference of experts in all of them. The preferred option, which is again is clearly superior to the others, is R9.5, "Increased investment in public and private R\&D, generating open, sustainable and inclusive innovation systems": the increase in resources (public and private) for more open innovation is perceived as an important aspect to exert progress on. The next one is R9.4, "Smart monitoring of pollutant emissions in industry and transport", presumably because the pollution is a critical challenge where the industry and transport have an important role to play. The third one is (R9.1), "AI for the generation of more efficient and sustainable structural engineering solutions", which suggests that AI may play an important role in improving the efficiency of infrastructure developments. The less preferred options, R9.3, "Digital transformation of the economy, through the development of Industry 4.0 and support for innovative startups", and R9.2, "AI to support the design of communication and transport infrastructure and networks", may have not had a higher number of favourable votes because they are less specific options than the other three.

Table 11. Proposed actions for SDG 9 involving the use of AI and digital technologies.

Goal 9: Industry, innovation and infrastructure.

R9.1-AI for the generation of more efficient and sustainable structural engineering solutions.

R9.2-AI to support the design of communication and transport infrastructure and networks.

R9.3-Digital transformation of the economy, through the development of Industry 4.0 and support for innovative startups.

R9.4-Smart monitoring of pollutant emissions in industry and transport.

R9.5-Increased investment in public and private R\&D, generating open, sustainable and inclusive innovation systems.

$$
P^{9}=\left[\begin{array}{ccccc}
0 & 0.56 & 0.5 & 0.43 & 0.1 \\
0.44 & 0 & 0.29 & 0.2 & 0.08 \\
0.5 & 0.71 & 0 & 0.14 & 0.09 \\
0.57 & 0.8 & 0.86 & 0 & 0.1 \\
0.9 & 0.92 & 0.91 & 0.9 & 0
\end{array}\right] ; C P^{9}=0.88 ; T E^{9}=193
$$

\begin{tabular}{|c|c|c|c|c|c|}
\hline & R9.1 & R9.2 & R9.3 & R9.4 & R9.5 \\
\hline QGDD & 0.48 & 0.27 & 0.38 & 0.72 & 0.91 \\
\hline QGNDD & 0.94 & 0.54 & 0.71 & 1.0 & 1.0 \\
\hline Not Known & 0 & 0 & 0 & 0 & 0 \\
\hline
\end{tabular}

$R 9.5 \succ R 9.4 \succ R 9.1 \succ R 9.3 \succ R 9.2$ 


\subsection{SDG 10: Reduced Inequalities}

Considering this goal focused on reducing inequalities, we have presented four different proposals (see Table 12). The most relevant one was R10.4, "Support for small and medium enterprises in digital resources for globalization and competitive advantage". This might be because globalization and e-commerce buttress big enterprises, putting small and medium enterprises in a relatively disadvantaged position against big companies. The second preferred proposal was R10.2, "Smart technologies to promote non-discriminatory citizen participation" because social participation is considered relevant for a healthy and fair society. The third one is R10.3, "Analysis of social polarization in social networks to combat discriminatory behaviors". Finally, the clearly less important alternative according to the respondents was R10.1, "Personalized digital banking against the economic breach". Since the importance and potential role of digital banking in the future may not be clear yet, or because the niche of established users of digital banking applications is still not grown enough, this paradigm might not have gained sufficiently popularity and consolidation yet as a service amongst the public.

Table 12. Proposed actions for SDG 10 involving the use of AI and digital technologies.

Goal 10: Reduced inequalities.

R10.1-Personalized digital banking against the economic breach.

R10.2 - Smart technologies to promote non-discriminatory citizen participation.

R10.3-Analysis of social polarization in social networks to combat discriminatory behaviors.

R10.4-Support for small and medium enterprises in digital resources for globalization and competitive advantage.

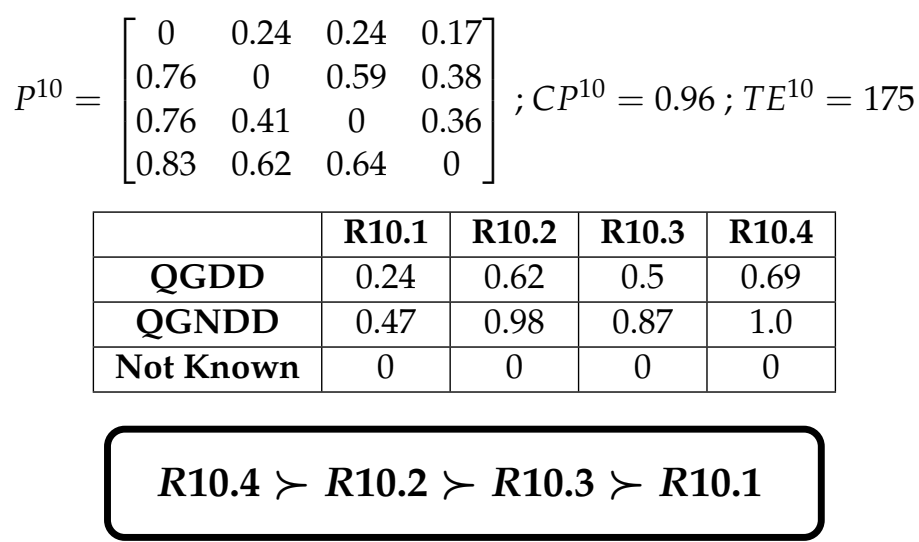

\subsection{SDG 11: Sustainable Cities and Communities}

There are five proposed courses of action to pursue this SDG aimed at building inclusive, safe, resilient and sustainable urban environments (presented in Table 13). In this case, there are two strongly favorite alternatives against the other three: (i) R11.3, focused on the potential benefits of machine learning algorithms to manage citizens' data and support decisions upon large volumes of unified urban data; followed by (ii) R11.5, which entails the joint use of robots, sensor infrastructures and drones to guarantee safe cities, namely by implementing urban resilience principles, as well as AI-driven protocols for disaster management, prevention and recovery. A moderate preference is shown towards R11.1, which proposes the use of digital twins for better planning and development of services in a city. By contrast, there was a clear lower preference towards virtual and augmented reality services as an alternative approach for enacting such services (R11.2), thereby hinting at a majority opinion that leans towards digital twins as a more promising solution to this end. Lastly, the transparency and security offered by blockchain (R11.4) to enhance electronic processes such as payments, is perceived as the least preferred of the proposed actions under this SDG, despite the proved popularity of this technology for other SDGs. This is a clear example of how people's perceptions about the role of digital 
technologies for the SDGs can greatly vary depending on the nature and characteristic of the goal in question.

Table 13. Proposed actions for SDG 11 involving the use of AI and digital technologies.

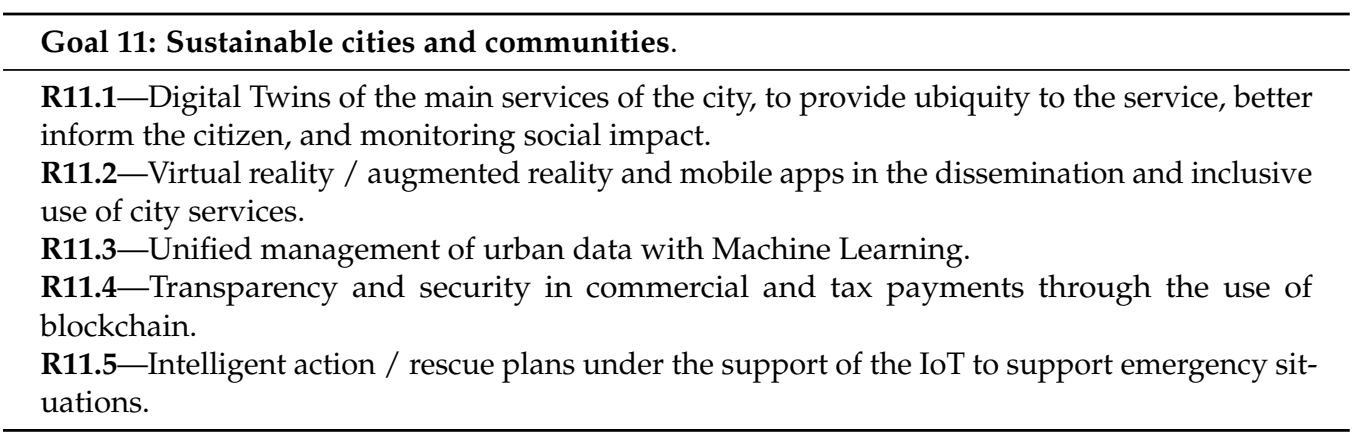

\begin{tabular}{|c|c|c|c|c|c|c|}
\hline \multirow{5}{*}{$P^{11}=$} & 0.69 & 0.22 & 0.630. & \multirow{5}{*}{\multicolumn{3}{|c|}{; $C P^{11}=0.9 ; T E^{11}=1$}} \\
\hline & 0.31 & 0.12 & $0.54 \quad 0$ & & & \\
\hline & $0.78 \quad 0.88$ & 0 & 0.920 & & & \\
\hline & $0.37 \quad 0.46$ & 0.08 & 0. & & & \\
\hline & \multirow[t]{2}{*}{0.95} & 0.47 & 0.78 & & & \\
\hline & & R11.1 & R11.2 & R11.3 & R11.4 & R11.5 \\
\hline & QGDD & 0.47 & 0.33 & 0.84 & 0.32 & 0.76 \\
\hline & QGNDD & 0.77 & 0.65 & 1.0 & 0.64 & 1.0 \\
\hline & Not Known & 0 & 3 & 1 & 0 & 1 \\
\hline \multicolumn{7}{|c|}{$R 11.3 \succ R 11.5 \succ R 11.1 \succ R 11.2 \sim R 11.4$} \\
\hline
\end{tabular}

\subsection{SDG 12: Responsible Production and Consumption}

The results of voting on the five alternatives proposed to help achieving this goal (see Table 14) report a strict order of preference across all of them, with roughly equidistant positions between each one in terms of dominance degrees (QGDD). Accordingly, R12.2 which advocates the Big Data and AI binomial to study citizens' consumption patterns and optimally adapt production processes to them, constitutes the most popular proposal, arguably because it jointly considers the two pillars that characterize this SDG. IoT and sensorization of production processes and consumption habits (R1.1) also received positive attention. R12.5 is related to decision support, recommender systems and blockchain for optimal, more sustainable and transparent producer-consumer chains and it is established as an intermediately preferred solution, whereas the two least preferred solutions were: $\mathbf{R} 12.3$ on the utilization of digital twins for sustainability testing in production processes; and R12.4 about augmented reality to raise awareness on sustainability in final consumers. A possible reason for these two options having received less votes could be the fact that each of them addressed only one of the two sides of the production-consumption binomial, whereas the other three alternatives holistically reflect the existing interaction between both pillars.

\subsection{SDG 13: Climate Action}

The proposed actions for SDG 13 are shown in Table 15. The results reveal a preference on R13.1 over the rest. This recommendation advocates the use of advanced AI techniques for natural disaster prediction. This is followed by R13.2 and R13.4 with similar dominance degrees, dealing with the adoption of sensing and IoT techniques for early-warning natural hazards monitoring and the development of AI models for urban traffic management optimisation, respectively. The next followed recommendation is R13.3 referring to the 
extension of pre-learnt AI models to other regions of the world (possibly least developed countries) thus creating worldwide holistic predictive models for natural hazard prevention. The least followed recommendation with a significant difference in the degree of dominance over the rest is R13.3, which stands for the use of augmented reality and conversational agents to improve environmental education. This may arguably be interpreted as a less immediate environmental priority compared to pressing issues such as disaster prevention and urban pollution mitigation.

Table 14. Proposed actions for SDG 12 involving the use of AI and digital technologies.

Goal 12: Responsible consumption and production.

R12.1-IoT technologies in production factories, supply networks and common places of consumption for greater sensorization of production processes and consumption habits.

R12.2 - Big data and artificial intelligence on data series of production processes and consumption patterns.

R12.3-Digital twins that allow the study of sustainability and "virtual" experimentation on production processes.

R12.4-Augmented reality to inform consumers of sustainability aspects related to the products they consume.

R12.5-Decision support systems, recommender systems and blockchain to improve sustainability in production processes and greater transparency in producer-consumer communication.

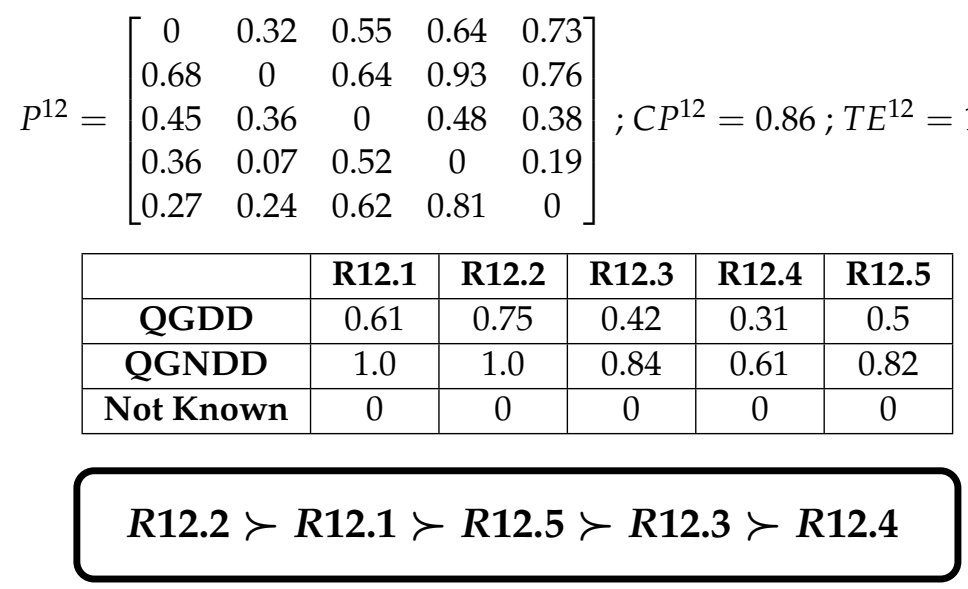

Table 15. Proposed actions for SDG 13 involving the use of AI and digital technologies.

Goal 13: Climate action.

R13.1-Improve natural disaster prediction using advanced AI models.

R13.2-IoT sensor technologies to allow AI to make predictions of natural disasters in real time.

R13.3 - Creation of predictive models worldwide, applying pre-learnt models in different areas of the world.

R13.4-AI to optimize urban traffic, thereby reducing pollution, through improvements in traffic signs.

R13.5-Augmented Reality and Conversational Agents to improve Environmental education.

$$
P^{13}=\left[\begin{array}{ccccc}
0 & 0.65 & 0.69 & 0.53 & 0.79 \\
0.35 & 0 & 0.54 & 0.54 & 0.93 \\
0.31 & 0.46 & 0 & 0.42 & 0.88 \\
0.47 & 0.46 & 0.58 & 0 & 0.82 \\
0.21 & 0.07 & 0.12 & 0.18 & 0
\end{array}\right] ; C P^{13}=0.92 ; T E^{13}=180
$$




\begin{tabular}{|c|c|c|c|c|c|}
\hline & R13.1 & R13.2 & R13.3 & R13.4 & R13.5 \\
\hline QGDD & 0.68 & 0.58 & 0.49 & 0.56 & 0.16 \\
\hline QGNDD & 1.0 & 1.0 & 0.9 & 0.98 & 0.32 \\
\hline Not Known & 0 & 0 & 0 & 0 & 0 \\
\hline
\end{tabular}

\section{$R 13.1 \succ R 13.2 \sim R 13.4 \succ R 13.3 \succ R 13.5$}

\subsection{SDG 14: Life Below Water}

From the recommendations given for this SDG (see Table 16) we can observe a clear preference for R14.1 against the rest. This recommendation stands for the adoption of advanced technologies to detect and monitor the expansion of plastic litter in the open seas. This is becoming a pressing global environmental issue with more than 10 million tonnes of plastic dumped into the sea annually [28], thereby being critically perceived by the respondents. The second most preferred option (R14.2) stands for the expansion of ocean monitoring technologies so that decisions on preservation and resources exploitation can be rationally made based on scientific information. The least followed recommendation is R14.3 which considers the adoption of blockchain technology to secure sensitive oceanic data and information so as to prevent criminal activities such as illegal fishing and over-fishing.

Table 16. Proposed actions for SDG 14 involving the use of AI and digital technologies.

Goal 14: Life below water.

R14.1-Technologies for detection, quantification and monitoring of marine plastic debris with a wide range of action.

R14.2-Low-cost, highly reliable ocean monitoring technologies.

R14.3 - Blockchain technology for the secure management of information systems and management of oceanic ecosystems.

$$
P^{14}=\left[\begin{array}{ccc}
0 & 0.77 & 0.98 \\
0.23 & 0 & 0.89 \\
0.02 & 0.11 & 0
\end{array}\right] ; C P^{14}=0.91 ; T E^{14}=180
$$

\begin{tabular}{|c|c|c|c|}
\hline & R14.1 & R14.2 & R14.3 \\
\hline QGDD & 0.9 & 0.63 & 0.07 \\
\hline QGNDD & 1.0 & 0.78 & 0.15 \\
\hline Not Known & 0 & 0 & 0 \\
\hline
\end{tabular}

$$
R 14.1 \succ R 14.2 \succ R 14.3
$$

\subsection{SDG 15: Life on Land}

The result of applying the decision making model against the alternatives presented in Table 17 shows that the recommendation of using AI to optimize water consumption in crops (R15.3) dominates over the rest of recommendations. The results also show that R15.2 (AI to detect diseases in crops) and R15.5 (Big Data to efficiently integrate and analyze data) are equally preferred since their QGDD and QGNDD are very similar even though both recommendations are someway dissimilar in terms of methods and overall impact. Besides, the results show that the use of intelligent sensors and IoT for real-time detection in applications like fire action (R15.4) has received lower attention. Contrary to other SDGs' recommendations related to IoT with very favourable results, this outcome is surprising since such a technology, even when still needs development to reach greater level of maturity, has demonstrated a strong efficiency and impact on fire detection [29-31] and land health monitoring [32-34]. Notwithstanding, it is important to remark that this 
result should be interpreted in terms of relative, pairwise preferences among the five candidate recommendations.

Table 17. Proposed actions for SDG 15 involving the use of AI and digital technologies.

\section{Goal 15: Life on Land.}

R15.1-AI for prediction and early detection of fires using aerial images and/or sensors

R15.2-AI for early detection of diseases in crops and reducing losses

R15.3-AI to optimize water consumption in crops

R15.4-Incorporation of intelligent sensors with IoT technologies for intelligent detection in real time

R15.5 - Big Data to efficiently integrate and analyze the data collected in large areas

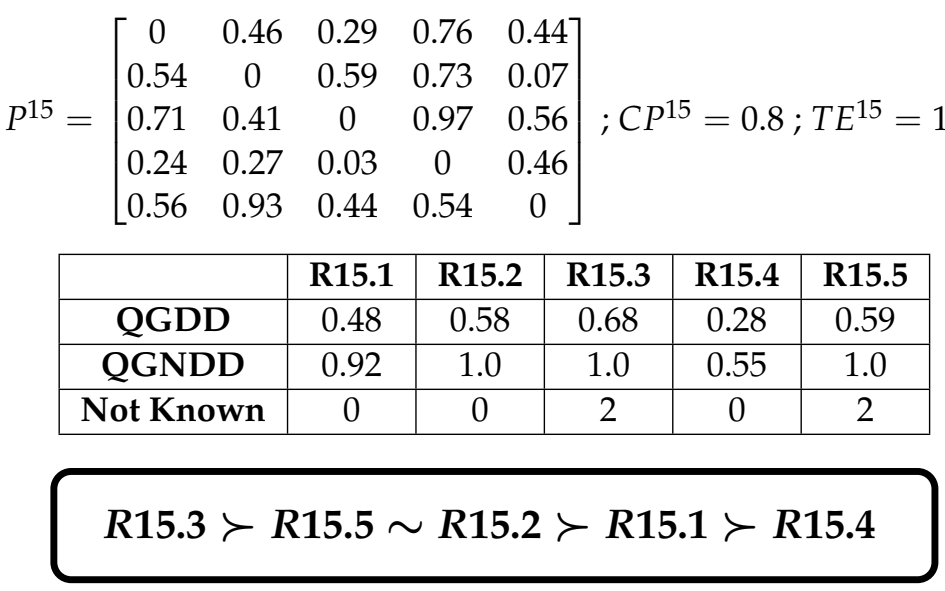

\subsection{SDG 16: Peace, Justice and Solid Institutions}

From the proposed alternatives for this SDG (see Table 18) and the results of the application of the decision making process it is clear that there is a strict order of preference among the four options, with a clear dominance of R16.2 over the rest. This recommendation stands for the use of the Blockchain to prevent institutional corruption, which is a solution being successfully tested in applications of corporate government and cryptocurrency transactions, to cite some of the most well-known ones. From the three remaining options, the recommendation R16.4, which advocates the use of expert systems for judicial processing as support tools, received positive attention. From the authors' perspective, the higher dominance of R16.2 and R16.4 over the rest might be due to the fact that they constitute feasible technologies which already have demonstrated a strong potential on these applications. In addition, they are based on clear and objective methods known by the AI community, as opposed to R16.1 and R16.3 whose technology foundations might have induced some uncertainty over the respondents, even though solutions like the ones given in $\mathbf{R} 16.3$ are successfully applied nowadays in several countries.

Table 18. Proposed actions for SDG 16 involving the use of AI and digital technologies.

\footnotetext{
Goal 16: Peace, justice and solid institutions.

R16.1-Machine Learning as a support tool for anticipating crime incidence and improving security response

R16.2-Safe and reliable institutions through the use of Blockchain to prevent institutional corruption, both publicly and privately

R16.3-Combination of AI techniques with other technological solutions (drones, IoT, etc.) as integrated tools for the fight against crime

R16.4-Self-adaptive expert systems for judicial processing as support for the integration of the judicial system for citizens
} 


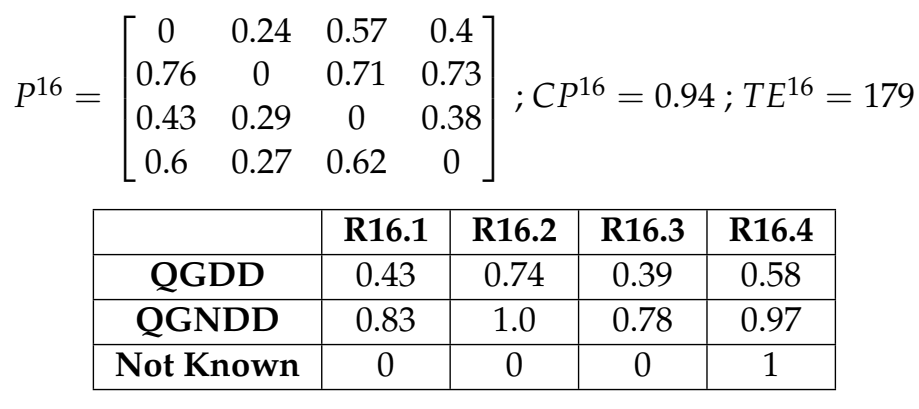

\section{$R 16.2 \succ R 16.4 \succ R 16.1 \succ R 16.3$}

\subsection{SDG 17: Partnerships for the Goals}

For the last -and arguably the most transversal- of the 17 SDGs, there is a highly consistent preference relation reflecting the social opinion on the four proposes alternatives (see Table 19). By observing both QGDD and QGNDD, there is overall a clear distinction between three positively rated alternatives (R17.1, R17.2 and R17.4) against one alternative that exhibits less preference when compared to the aforesaid three (R17.3). On the opposite end of the spectrum, R17.4 outranks the rest of proposals: this suggests that societies have a strong interest in witnessing a greater engagement by governments in building comprehensive programs, partnerships and frameworks to bring AI and digital technologies into all aspects of people's daily lives, in order to ultimately align such lives with sustainable development. Meanwhile, there is a similar degree of interest in two closely interrelated proposals, R17.1 and R17.2. These solutions concern about building interdisciplinary alliances and standards founded on ethical considerations of AI, and developing systematic codes of practice for using AI technologies across professional sectors, respectively. The lower preference for R17.3 (despite the importance of enabling large-scale data collection and sharing infrastructure from the authors' point of view [5]) could indicate that citizens might be concerned about the use(s) that may be given to collected data associated to themselves. Thus, it is remarkably important to enforce mechanisms that not only safeguard data protection and privacy, but also transmits a sense of reliability to the citizens.

Table 19. Proposed actions for SDG 17 involving the use of AI and digital technologies.

\section{Goal 17: Partnerships for the goals.}

R17.1-Alliances for AI and digital technologies based on ethical principles and multidisciplinary experts.

R17.2 - Codes of professional practice in organizational and professional environments under the use of AI and digital technologies.

R17.3-Drive global alliances in the widespread use of economically sustainable data collection.

R17.4 - Carry out government efforts to bring AI and digital technologies to the whole of society.

$$
P^{17}=\left[\begin{array}{cccc}
0 & 0.48 & 0.71 & 0.41 \\
0.52 & 0 & 0.67 & 0.43 \\
0.29 & 0.33 & 0 & 0.28 \\
0.59 & 0.57 & 0.72 & 0
\end{array}\right] ; C P^{17}=0.98 ; T E^{17}=180
$$

\begin{tabular}{|c|c|c|c|c|}
\hline & R17.1 & R17.2 & R17.3 & R17.4 \\
\hline QGDD & 0.54 & 0.55 & 0.3 & 0.62 \\
\hline QGNDD & 0.97 & 0.99 & 0.59 & 1.0 \\
\hline Not Known & 1 & 0 & 1 & 0 \\
\hline
\end{tabular}

$R 17.4 \succ R 17.2 \sim R 17.1 \succ R 17.3$ 


\section{Global Results \& Lessons Learnt}

Once we have applied the decision making process for each of the SDGs and their associated recommendations, the use of the different AI techniques and related digital technologies are analyzed to establish those which deserve more attention and investment for the purpose of reaching the SDGs. To this end, for each considered AI technique and digital technology, some SDGs are selected under the following criteria: (i) such technology is highlighted in a recommendation that has been ranked in the first or second place; and (ii) its associated QGDD is greater than 0.5 .

Figure 3 depicts the results on the use of the digital technologies and reveals that explainable and trustworthy AI along with IoT are some of the preferred technologies to tackle many different SDGs. Moreover, it is shown that these techniques are better ranked in regards to the economic, social, and environmental dimensions of the SDGs. Regarding explainable and trustworthy AI, it is manifested that this technology has revolutionized many different disciplines and it may also be well used in order to fulfill the SDGs thanks to its analysis and prediction capabilities. IoT on its side allows a much extensive and detailed sensorization that can be used to feed data into information systems. That information is a valuable asset that can be later analyzed to make better decisions, improve production processes, predict changes and failures and continuously supervise the evolution of every indicator of the evolution of the SDGs.

Big data processing algorithms have also been preferred by the respondents. Its is clear that the amount of information that is currently gathered almost for any discipline requires great efforts to be properly processed and analyzed. Furthermore, the experts have expressed that this technology might be specially interesting for the economic and environmental dimensions of sustainability ( "no proverty", "no hunger", "responsible consumption and production" and "life on land"). Blockchain has also been noted as an interesting technology due to its potential applications regarding governability and its distributed approach and data integrity characteristics in the "no poverty", "decent work and economic growth" and "peace, justice and strong institutions" SDGs.

Machine learning has been one of the preferred technologies for the social and environmental dimensions of the SDGs, probably due to its capacity to build models from data, which for the case of the social dimension, are difficult to create by hand due to the uncertainty in the variables related to human behaviour. Recommender and information systems have been also preferred technologies for some SDGs as they provide powerful tools that can help decision makers to create new policies based on the available data.

Finally, digital twins and computer vision have also been selected as preferred technologies according to the respondents for some of the SDGs. Note that these technologies are more specific tools than some of the previously presented ones, thus this might be the reason to explain why the respondents do not consider them as equally interesting as others.

Some other general lessons that can be extracted from the results are:

- Out of 3 SDGs focused on equality, only "quality education" received highly preferred recommendations (with QGDD greater than 0.7), which means that those recommendations are particularly worth investing in against the other ones within a given goal. On the other side, "gender equality" and "reduced inequalities" do not present a so clear preference for none of their proposed recommendations, which implies that a variety of different AI related technologies should be considered in order to fulfill these goals.

- Regarding R8.1, which is the most strongly preferred option in SDG 8 ("decent work and economic growth") and one of the most positively valued recommendations overall, it is worth noting that it also has a clear strong relationship with the improvement of sustainable, equal, inclusive and digital-driven education systems, i.e., SDG 4.

- Both SDGs 9 ("Industry, Innovation and Infrastructure") and 14 ("Life Below Water") present both highly voted and low-voted recommendations, i.e., a clearly skewed distribution of pairwise votes: this is intuitively an indicator of high consensus among 
participants' opinions on the alternatives in these two SDGs, with clear majorities leaning towards highly voted recommendations.

- On the contrary, in the Economic dimension and life perspective as a whole (SDGs 1-3) there is only one highly ranked recommendation (R3.1) and no low-ranked recommendations at all, which means that almost all recommendations are positioned in intermediate ranking values and therefore there are balanced views on the potential value for all of them, with little signs of strong preference for (or against) none of them.

- The respondents have clearly expressed the potential value of IoT in the environmental dimension in which the fulfillment of many SDGs depends on strict observance on variables as pollution, use of natural resources and so on.

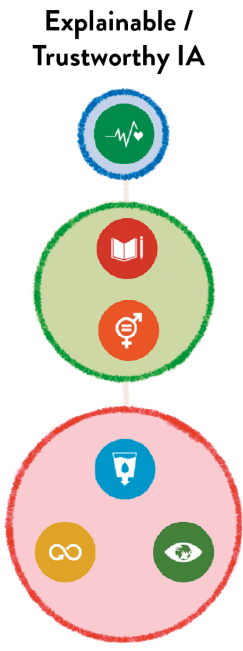

Blockchain

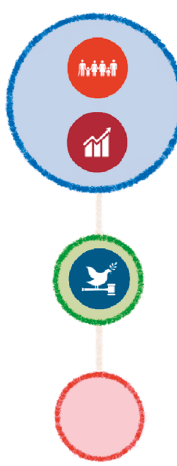

Digital Twin

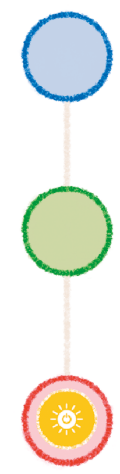

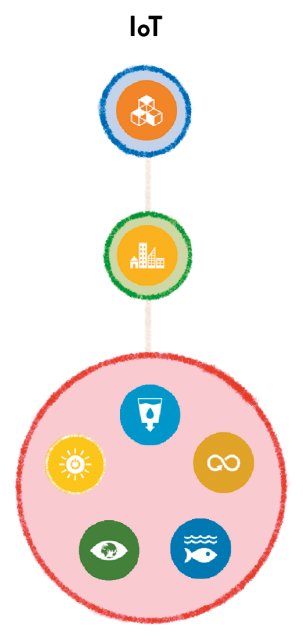

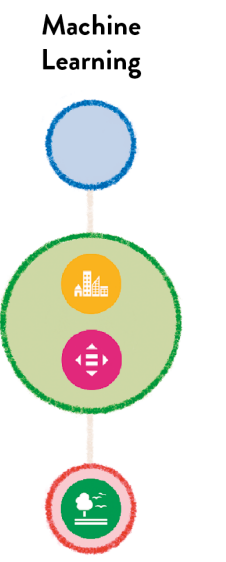

Computer Vision

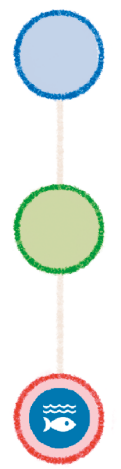

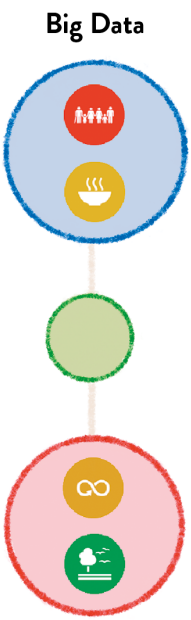

Recommender and Information Systems

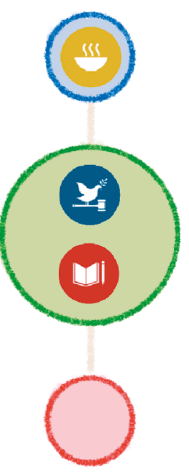

Economic dimension

Social dimension

Environmental dimension

Figure 3. AI techniques and related digital technologies that are used in the preferred recommendations for the different SDGs. 


\section{Conclusions}

In this paper a novel group decision making process to rank several recommendations about the use of AI and related digital technologies towards the achievement of the United Nations' SDGs, was presented. These recommendations were obtained in a previous study [26] which analyzed the specialized literature to track the current trends in the application of those technologies in the SDGs field. As a result, for each of the SDGs, a ranking of recommendations is provided which can be used by decision makers (which may not be experts in AI and its related technologies) as a guide to state which digital technologies are more promising to invest on in order to fulfill each of the goals.

The decision making process is based on surveys that have been responded by experts in the fields of AI and digital technologies as well as experts in the different SDGs. The surveys have been carried out by using an online tool that eases the expression of preferences by means of simple comparisons avoiding the use of numerical or linguistic scales that can be difficult to use for respondents that are not used to them. Moreover, the online tool allows the expression of uncertainty when the respondents do not have a clear opinion about the presented alternatives or does not have enough knowledge about some of them.

The presented rankings of the recommendations are comparative. Therefore, a recommendation which has been ranked as the last one must not be considered a "bad one". It is only that there are other recommendations that are preferred.

From the 17 rankings we have analyzed which particular digital technologies are among the most mentioned in the best ranked recommendations. In that way we have concluded that almost all the presented AI techniques and related digital technologies are seen as relevant for at least some of the SDGs (depending on the nature of the particular SGD).

As overall concluding remark, we can state that future AI and other digital and emerging technologies act as enablers of the SDGs. More particularly,

- Recommendations on the use of AI and other digital technologies are drawn from a comprehensive analysis of the current literature, which reflects on how these digital technologies and AI can lead to fundamental solutions to advance the SDGs;

- It has been evidenced the importance of the views of individuals, many of them experts on a given SDG, who participate in an open consultation by contributing their knowledge on the topic;

- The analysis of perceived importance in developing solutions has highlighted that both $\mathrm{AI}$ and its associated digital technologies are key tools for advancing AI-based solutions that pursue the SDGs.

From the study we can also remark that:

- IoT proves to be a highly recommended technology along with explainable AI and Big Data for the achievement of the SDGs.

- Blockchain proves to have it highest popularity as a potential tool to enforce reliable, fair and trustworthy institutions and governments.

- Many of the presented AI related technologies have been considered as the preferred ones for some of the SDGs. This signifies that this whole family of technologies are worth being invested in to improve the chances of fulfilling the SDGs.

Finally, we want to emphasize that AI and its related technologies are currently a driving force in many different fields and therefore it is important that governments, companies, organizations and citizens recognize their potential and utility to reach the SDGs in the following years. Moreover, as those technologies are mainly based on data, it is also very important to invest on data infrastructures that allow the gathering, custody, transformation and accessibility of high quality data sets to enable AI-based solutions to help in the attainment of the SDGs.

Author Contributions: Conceptualization: S.A., R.M. (Rosana Montes), D.M., I.P., E.M.-C., M.C., J.C., F.J.M., F.H., B.F., C.M., R.M. (Rosario Marchena), J.P.d.V.; Investigation: S.A., R.M. (Rosana Montes), D.M., I.P., E.M.-C., M.C., J.C., F.J.M., F.H.; Funding acquisition: B.F., C.M., R.M. (Rosario Marchena), 
J.P.d.V.; Methodology: S.A., R.M. (Rosana Montes), D.M., I.P., E.M.-C., M.C., J.C., F.J.M., F.H.; Software: S.A., R.M. (Rosana Montes), D.M., I.P., E.M.-C., M.C., J.C., F.J.M., F.H.; Writing-original draft: S.A., R.M. (Rosana Montes), D.M., I.P., E.M.-C., M.C., J.C., F.J.M., F.H.; Writing-review \& editing: S.A., R.M., D.M., I.P., E.M.-C., M.C., J.C., F.J.M., F.H., B.F., C.M., R.M. (Rosario Marchena), J.P.d.V.; Original illustrations from P.G.-M. All authors have read and agreed to this version of the manuscript.

Funding: This work was supported by the contract OTRI-4408 between the University of Granada and the Royal Academy of Engineering of Spain financed by Ferrovial S.A. Eugenio Martínez Cámara was supported by the Spanish Government fellowship programme Juan de la Cierva Incorporación (IJC2018-036092-I).

Data Availability Statement: All raw data regarding the surveys in this paper and the python scripts to process it can be found in https://github.com/ari-dasci/S-SDG-Decision (accesed date: 25 May 2021).

Conflicts of Interest: The authors declare no conflict of interest.

\section{Abbreviations}

The following abbreviations are used in this manuscript:

$\begin{array}{ll}\text { AI } & \text { Artificial Intelligence } \\ \text { IoT } & \text { Internet of Things } \\ \text { SDGs } & \text { Sustainable Development Goals } \\ \text { QGDD } & \text { Quantifier Guided Dominance Degree } \\ \text { QGNDD } & \text { Quantifier Guided Non Dominance Degree }\end{array}$

\section{References}

1. United Nations. Transforming Our World: The 2030 Agenda for Sustainable Development; UN General Assembly, United Nations: New York, NY, USA, 2015.

2. Ripley, B.D. Pattern Recognition and Neural Networks; Cambridge University Press: Cambridge, UK, 1996. [CrossRef]

3. Lohr, S. From Agriculture to Art-The A.I. Wave Sweeps In. New York Times, 19 October 2018; Section F, Page 7. Available online: https:/ /www.nytimes.com/2018/10/21/business / from-agriculture-to-art-the-ai-wave-sweeps-in.html (accessed on 25 May 2021).

4. Bonilla, S.; Silva, H.; da Silva, M.; Gonçalves, R.; Sacomano, J. Industry 4.0 and Sustainability Implications: A Scenario-Based Analysis of the Impacts and Challenges. Sustainability 2018, 10, 3740. [CrossRef]

5. Palomares, I.; Martínez-Cámara, E.; Montes, R.; García-Moral, P.; Chiachio, M.; Chiachio, J.; Alonso, S.; Melero, F.; Molina, D.; Fernández, B.; et al. A Panoramic View and SWOT Analysis of Artificial Intelligence for Achieving the Sustainable Development Goals: Progress and Prospects. Appl. Intell. 2021, in press.

6. Wu, J.; Guo, S.; Huang, H.; Liu, W.; Xiang, Y. Information and Communications Technologies for Sustainable Development Goals: State-of-the-Art, Needs and Perspectives. IEEE Commun. Surv. Tutor. 2018, 20, 2389-2406. [CrossRef]

7. Vinuesa, R.; Azizpour, H.; Leite, I.; Balaam, M.; Dignum, V.; Domisch, S.; Felländer, A.; Langhans, S.; Tegmark, M.; Nerini, F. The role of artificial intelligence in achieving the Sustainable Development Goals. Nat. Commun. 2020, 11, 1-10.

8. McCarthy, J.; Minsky, M.; Rochester, N.; Shannon, C.E. A Proposal for the Dartmouth Summer Research Project on Artificial Intelligence, August 31, 1955. AI Mag. 2006, 27, 12-14.

9. Russell, S.; Norvig, P. Artificial Intelligence: A Modern Approach, 4th ed.; Pearson: Harlow, UK, 2020.

10. Bublitz, F.M.; Oetomo, A.; Sahu, K.S.; Kuang, A.; Fadrique, L.X.; Velmovitsky, P.E.; Nobrega, R.M.; Morita, P.P. Disruptive Technologies for Environment and Health Research: An Overview of Artificial Intelligence, Blockchain, and Internet of Things. Int. J. Environ. Res. Public Health 2019, 16, 3847. [CrossRef] [PubMed]

11. Kshetri, N. The emerging role of Big Data in key development issues: Opportunities, challenges, and concerns. Big Data Soc. 2014, 1, 2053951714564227. [CrossRef]

12. Herrera-Viedma, E.; Palomares, I.; Li, C.C.; Cabrerizo, F.J.; Dong, Y.; Chiclana, F.; Herrera, F. Revisiting Fuzzy and Linguistic Decision Making: Scenarios and Challenges for Making Wiser Decisions in a Better Way. IEEE Trans. Syst. Man Cybern. Syst. 2021, 51, 191-208. [CrossRef]

13. Seo, F.; Sakawa, M. Fuzzy multiattribute utility analysis for collective choice. IEEE Trans. Syst. Man Cybern. 1985, SMC-15, 45-53. [CrossRef]

14. Tanino, T. On group decision making under fuzzy preferences. In Multiperson Decision Making Using Fuzzy Sets and Possibility Theory; Kacprzyk, J., Fedrizzi, M., Eds.; Kluwer: Norwell, MA, USA, 1990; pp. 172-185.

15. Kacprzyk, J. Group decision making with a fuzzy linguistic majority. Fuzzy Sets Syst. 1986, 18, 105-118. [CrossRef]

16. Chiclana, F.; Herrera, F.; Herrera-Viedma, E. Integrating multiplicative preference relations in a multipurpose decision-making model based on fuzzy preference relations. Fuzzy Sets Syst. 2001, 122, 277-291. [CrossRef] 
17. Herrera-Viedma, E.; Herrera, F.; Chiclana, F. A consensus model for multiperson decision making with different preference structures. IEEE Trans. Syst. Man Cybern. Part A Syst. Hum. 2002, 32, 394-402. [CrossRef]

18. Chiclana, F.; Herrera, F.; Herrera-Viedma, E. A note on the internal consistency of various preference representations. Fuzzy Sets Syst. 2002, 131, 75-78. [CrossRef]

19. Orlovsky, S. Decision-making with a fuzzy preference relation. Fuzzy Sets Syst. 1978, 1, 155-167. [CrossRef]

20. Alonso, S.; Chiclana, F.; Herrera, F.; Herrera-Viedma, E.; Alcala-Fdez, J.; Porcel, C. A consistency based procedure to estimate missing pairwise preference values. Int. J. Intell. Syst. 2008, 23, 155-175. [CrossRef]

21. Alonso, S.; Herrera-Viedma, E.; Chiclana, F.; Herrera, F. Individual and Social Strategies to Deal with Ignorance Situations in Multi-Person Decision Making. Int. J. Inf. Technol. Decis. Mak. 2009, 8, 313-333. [CrossRef]

22. Herrera-Viedma, E.; Chiclana, F.; Herrera, F.; Alonso, S. Group Decision-Making Model With Incomplete Fuzzy Preference Relations Based on Additive Consistency. IEEE Trans. Syst. Man Cybern. Part B (Cybern.) 2007, 37, 176-189. [CrossRef]

23. Alonso, S.; Cabrerizo, F.; Chiclana, F.; Herrera, F.; Herrera-Viedma, E. An Interactive Decision Support System Based on Consistency Criteria. Mult. Valued Log. Soft Comput. 2008, 14, 371-386.

24. Salganik, M.; Levy, K. Wiki Surveys: Open and Quantifiable Social Data Collection. PLoS ONE 2015, 10, e0123483. [CrossRef]

25. Thurstone, L.L. The method of paired comparisons for social values. J. Abnorm. Soc. Psychol. 1927, 21, 384-400. [CrossRef]

26. Montes, R.; Melero, F.; Palomares, I.; Alonso, S.; Chiachío, J.; Chiachío, M.; Molina, D.; Martínez-Cámara, E.; Tabik, S.; Herrera, F. Inteligencia Artificial y Tecnologías Digitales para los ODS; Spain's Royal Academy of Engineering: Madrid, Spain, 2021.

27. Tanino, T. Fuzzy preference orderings in group decision making. Fuzzy Sets Syst. 1984, 12, 117-131. [CrossRef]

28. Jambeck, J.R.; Geyer, R.; Wilcox, C.; Siegler, T.R.; Perryman, M.; Andrady, A.; Narayan, R.; Law, K.L. Plastic waste inputs from land into the ocean. Science 2015, 347, 768-771. [CrossRef] [PubMed]

29. Huang, Z.; Liu, F. The Application of MAS towards Simulation of Forest Fire Spreading. Int. J. Multimed. Ubiquitous Eng. 2013, 8, 189-200. [CrossRef]

30. Kinaneva, D.; Hristov, G.; Raychev, J.; Zahariev, P. Early Forest Fire Detection Using Drones and Artificial Intelligence. In Proceedings of the 2019 42nd International Convention on Information and Communication Technology, Electronics and Microelectronics (MIPRO 2019_Proceedings), Opatija, Croatia, 20-24 May 2019; pp. 1060-1065.

31. Razavi-Termeh, S.; Sadeghi-Niaraki, A.; Choi, S.M. Ubiquitous GIS-Based Forest Fire Susceptibility Mapping Using Artificial Intelligence Methods. Remote Sens. 2020, 12, 1689. [CrossRef]

32. Mohd Kassim, M.; Mat, I.; Harun, A. Wireless Sensor Network in Precision Agriculture Application. In Proceedings of the 2014 International Conference on Computer, Information and Telecommunication Systems (CITS 2014), Jeju, Korea, 7-9 July 2014.

33. Nam, W.H.; Choi, J.Y.; Yoo, S.H.; Jang, M.W. A Decision Support System for Agricultural Drought Management Using Risk Assessment. Paddy Water Environ. 2012, 10, 197-207. [CrossRef]

34. Bhatnagar, V.; Chandra, R. IoT-Based Soil Health Monitoring and Recommendation System. In Internet of Things and Analytics for Agriculture; Springer Nature: Singapore, 2020; Volume 2, pp. 1-21. 